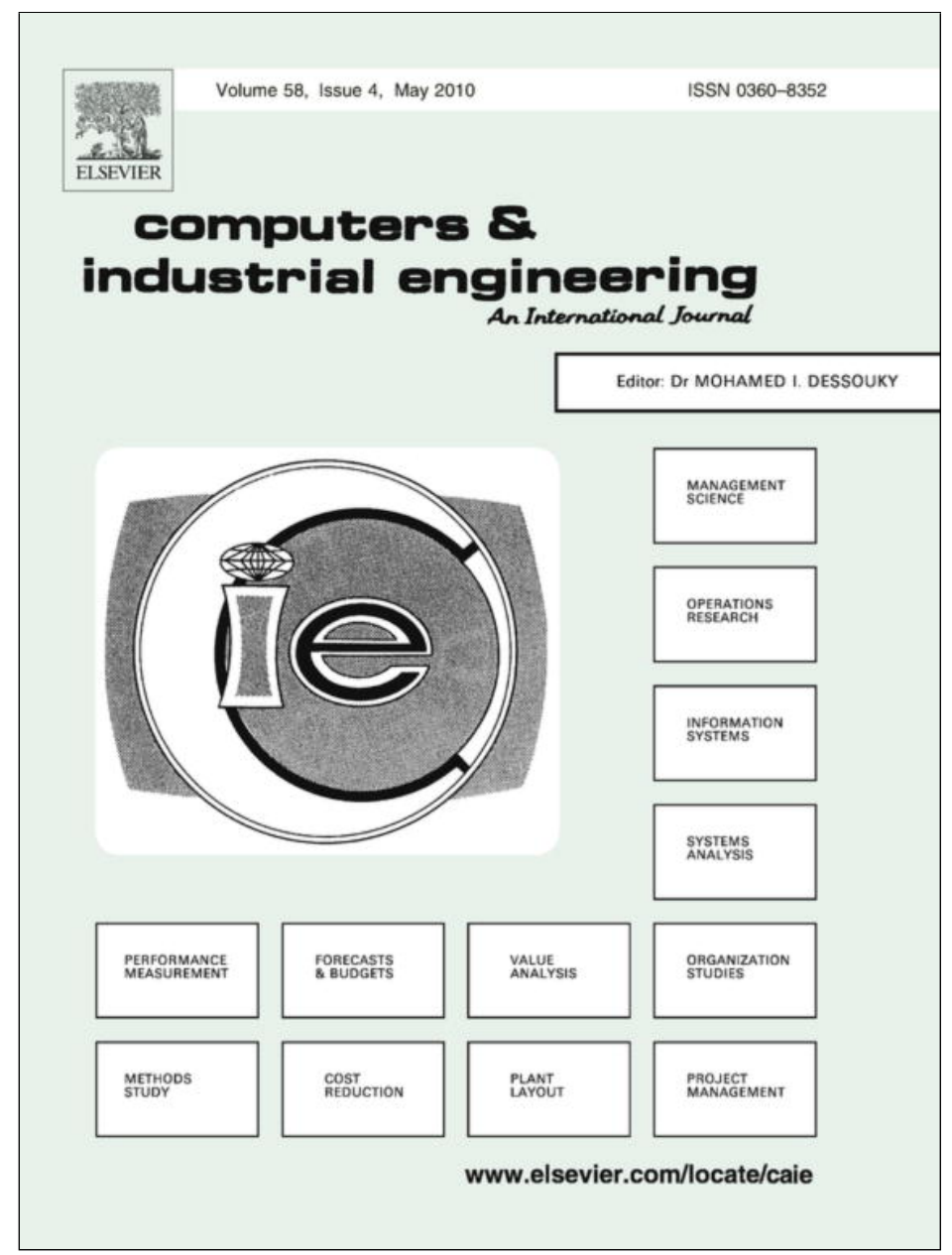

This article appeared in a journal published by Elsevier. The attached copy is furnished to the author for internal non-commercial research and education use, including for instruction at the authors institution and sharing with colleagues.

Other uses, including reproduction and distribution, or selling or licensing copies, or posting to personal, institutional or third party websites are prohibited.

In most cases authors are permitted to post their version of the article (e.g. in Word or Tex form) to their personal website or institutional repository. Authors requiring further information regarding Elsevier's archiving and manuscript policies are encouraged to visit:

http://www.elsevier.com/copyright 


\title{
Fuzzy decision making with immediate probabilities is
}

\author{
José M. Merigó* \\ Department of Business Administration, University of Barcelona, Av. Diagonal 690, 08034 Barcelona, Spain
}

\section{A R T I C L E I N F O}

Article history:

Received 16 May 2009

Received in revised form 20 October 2009

Accepted 8 January 2010

Available online 14 January 2010

\section{Keywords:}

Decision making

Immediate probabilities

OWA operator

Fuzzy numbers

Strategic selection

\begin{abstract}
A B S T R A C T
We developed a new decision-making model with probabilistic information and used the concept of the immediate probability to aggregate the information. This type of probability modifies the objective probability by introducing the attitudinal character of the decision maker. In doing so, we use the ordered weighting average (OWA) operator. When using this model, it is assumed that the information is given by exact numbers. However, this may not be the real situation found within the decision-making problem. Sometimes, the information is vague or imprecise and it is necessary to use another approach to assess the information, such as the use of fuzzy numbers. Then, the decision-making problem can be represented more completely because we now consider the best and worst possible scenarios, along with the possibility that some intermediate event (an internal value) will occur. We will use the fuzzy ordered weighted averaging (FOWA) operator to aggregate the information with the probabilities. As a result, we will get the Immediate Probability-FOWA (IP-FOWA) operator. We will study some of its main properties. We will apply the new approach in a decision-making problem about selection of strategies.

(c) 2010 Elsevier Ltd. All rights reserved.
\end{abstract}

\section{Introduction}

In the literature, we find a wide range of methods for decision making (Engemann, Filev, \& Yager, 1996; Engemann, Miller, \& Yager, 2004; Figueira, Greco, \& Ehrgott, 2005; Gil-Aluja, 1999; Merigó, 2008; Xu, 2009; Xu \& Yager, 2008; Yager, 1999; Yager \& Kacprzyk, 1997). A very common decision-making method is that which uses probabilities in its analysis. This method is known as decision making under risk environment. The use of probabilities permits an objective modelization of the decision-making problem and its uncertainties under analysis. However, although we can assess the problem in an objective way by using probabilities, we do not know which result will occur in the future. Therefore, sometimes we may prefer to consider a different form of modelization that is also endowed with probabilities.

In order to solve this problem (Engemann et al., 1996; Yager, Engemann, \& Filev, 1995), the concept of immediate probabilities was suggested. This method transforms the initial probabilities into new ones that consider the attitudinal character of the decision maker. The reason for doing so is that although the probabilistic information is objective, we still do not know exactly what is going to happen in the future. Therefore, although we can predict the outcome, the predictions are not guaranteed to be accurate. It is well known in statistics that if one performs only a single experiment, then it is difficult to accurately predict its result when

\footnotetext{
This manuscript was processed by Area Editor Mauro Birattari.

* Tel.: +34 9340219 62; fax: +34 934039882 .

E-mail address: jmerigo@ub.edu
}

using probabilities. However, if one were to perform the same experiment many times, then the results would be in accordance with the results predicted by the probabilities. The problem in decision making is that we are modeling a problem of the real life that will only occur once (or few times). Then, the probabilistic information may be affected by some exceptional situations, which are not probable. Therefore, it can be very useful to use immediate probabilities instead of the usual probabilities.

The use of immediate probabilities implies the use of the ordered weighted averaging (OWA) operator (Yager, 1988) in order to consider the attitudinal character of the decision maker. Then, although the decision maker considers the probabilistic information, he can manipulate it according to his degree of optimism or pessimism. The OWA operator provides a parameterized family of aggregation operators that includes the maximum, the minimum and the average operators. Since its appearance, it has been studied by many authors (Alonso, Cabrerizo, Chiclana, Herrera, \& Herrera-Viedma, 2009; Beliakov, Pradera, \& Calvo, 2007; Canós \& Liern, 2008; Kacprzyk \& Zadrozny, 2009; Merigó, 2008; Merigó \& Casanovas, 2009; Merigó \& Gil-Lafuente, 2008, 2009; Torra, 1997; Wang, Luo, \& Liu, 2007; Wu, Li, Li, \& Duan, 2009; Xu, 2005, 2009; Yager, 1993, 2007, 2008, 2009; Yager \& Filev, 1994; Yager \& Kacprzyk, 1997).

When using the OWA operator, it is assumed that the information can be represented with exact numbers. However, this may not be the real situation found in the specific decision-making problem at hand. Sometimes, the information is imprecise and it is necessary to use another approach to assess the information. In this type of problem, it can be useful to use fuzzy numbers 
(FNs). FNs (Chang \& Zadeh, 1972; Dubois \& Prade, 1980; Kaufmann \& Gupta, 1985; Zadeh, 1975) consider the best and worst possible scenarios, as well as an intermediate values there between, that may occur. In order to use the OWA operator in these situations, the adoption of the fuzzy OWA (FOWA) operator has been suggested (Merigó \& Casanovas, 2008; Mitchell \& Estrakh, 1998; Yager, 2008; Zarghami \& Szidarovszky, 2009; Zhou, Chiclana, John, \& Garibaldi, 2008).

The aim of this paper is to develop a new decision-making model by using immediate probabilities and information that can be represented with FNs. In doing so, we will suggest a new aggregation operator: the Immediate Probability-Fuzzy OWA (IP-FOWA) operator. This operator uses the OWA operator, FNs and probabilistic information. It is quite useful because it can assess the uncertain information within the problem by using both FNs and the probabilistic information that considers the attitudinal character of the decision maker. We study some of its main properties and distinguish between different particular cases such as the IP-median-FOWA, the IP-olympic-FOWA and the IP-centered-FOWA.

We also developed an application of the new approach in a decision-making problem about selection of strategies. The main advantage of using IP-FOWA operators is that we can consider a wide range of possible situations because the aggregation with OWAs permits us to consider a parameterized family of aggregation operators whose values are between the minimum and the maximum. Then, the decision maker will get a complete view of the decision problem while considering the probabilistic information and its attitudinal character. Note that in the literature, we find only a few studies about immediate probabilities (Engemann et al., 1996, 2004; Yager 1999). Moreover, by using FNs, we can assess in a more complete way the uncertain information of the decision problem because we are considering all possible results and able to consider the ones with the highest degree of possibility of occurrence. However, it is interesting to note that there are many potential applications that could be developed with them, for example in the actuarial sciences and statistics. More generally, we could consider that the immediate probabilities can be used in almost all problems already considered with the usual probabilities. Obviously though, its case-dependent usefulness will be more significant to the problem at hand.

This paper is organized as follows. In Section 2, we briefly review some basic concepts about FNs, the FOWA operator and the immediate probabilities. Section 3 introduces the IP-FOWA operator. In Section 4, we analyze different types of IP-FOWA operator. Section 5 illustrates the new approach with an example in decision making. Finally, in Section 6, we summarize the main conclusions of the paper.

\section{Preliminaries}

In this section, we briefly review the FNs, the fuzzy OWA operator and the concept of immediate probabilities.

\subsection{Fuzzy numbers}

The FN was introduced in (Chang \& Zadeh, 1972; Zadeh, 1975). Since then, it has been studied and applied by a wide array of authors, such as (Dubois \& Prade, 1980; Kaufmann \& Gupta, 1985).

A FN is a fuzzy subset (Zadeh, 1965) of a universe of discourse that is both convex and normal (Kaufmann \& Gupta, 1985). Note that the FN may be considered to be a generalization of the interval number (Moore, 1966).

In the literature, we find a wide range of FNs (Dubois \& Prade, 1980; Kaufmann \& Gupta, 1985). For example, a trapezoidal FN
(TpFN) $A$ of a universe of discourse $R$ can be characterized by a trapezoidal membership function $A=(\underline{a}, \bar{a})$ such that:

$\underline{a}(\alpha)=a_{1}+\alpha\left(a_{2}-a_{1}\right)$,

$\bar{a}(\alpha)=a_{4}-\alpha\left(a_{4}-a_{3}\right)$.

where $\alpha \in[0,1]$ and is parameterized by $\left(a_{1}, a_{2}, a_{3}, a_{4}\right)$ where $a_{1} \leqslant$ $a_{2} \leqslant a_{3} \leqslant a_{4}$, are real values. Note that if $a_{1}=a_{2}=a_{3}=a_{4}$, then the FN is a singleton, and if $a_{2}=a_{3}$, then the FN is represented by a triangular FN (TFN). Note that the TFN can be parameterized by $\left(a_{1}, a_{2}, a_{4}\right)$.

In the following, we review the FN arithmetic operations as follows. Let $A$ and $B$ be two TFN, where $A=\left(a_{1}, a_{2}, a_{3}\right)$ and $B=\left(b_{1}, b_{2}, b_{3}\right)$. Then:

(1) $A+B=\left(a_{1}+b_{1}, a_{2}+b_{2}, a_{3}+b_{3}\right)$.

(2) $A-B=\left(a_{1}-b_{3}, a_{2}-b_{2}, a_{3}-b_{1}\right)$.

(3) $A \times k=\left(k \times a_{1}, k \times a_{2}, k \times a_{3}\right)$; for $k>0$.

Note that other operations could be studied (Dubois \& Prade, 1980; Kaufmann \& Gupta, 1985) but in this paper we will focus on these ones.

\subsection{Fuzzy OWA operator}

The FOWA operator (Merigó, 2008; Mitchell \& Estrakh, 1998) represents an extension of the OWA operator. Essentially, its main difference is that the FOWA operator uses uncertain information in the arguments represented in the form of FNs. The reason for using this aggregation operator is that sometimes the available information cannot be assessed with exact numbers and it is necessary to use other techniques such as FNs. The FOWA operator provides a parameterized family of aggregation operators that include the fuzzy maximum, the fuzzy minimum and the fuzzy average criteria, among others.

Comparing FNs with interval numbers, we see that the FNs are more complete. This happens because they use a membership function to describe the possibility that an uncertain result will occur. Then, the FNs give the same information as the interval numbers, but they also explain the possibility that the internal values of the interval will occur. It can be defined as follows.

Definition 1. Let $\Psi$ be the set of fuzzy numbers. A FOWA operator of dimension $n$ is a mapping FOWA: $\Psi^{n} \rightarrow \Psi$ that has an associated weighting vector $W$ of dimension $n$ such that the sum of the weights is 1 and $w_{j} \in[0,1]$, then:

$\operatorname{FOWA}\left(\tilde{a}_{1}, \tilde{a}_{2}, \ldots, \tilde{a}_{n}\right)=\sum_{j=1}^{n} w_{j} b_{j}$,

where $b_{j}$ is the $j$ th largest of the $\tilde{a}_{i}$, and the $\tilde{a}_{i}$ are FN.

From a generalized perspective of the reordering step, we have to distinguish between the descending FOWA (DFOWA) operator and the ascending FOWA (AFOWA) operator. The weights of these operators are related by $w_{j}=w_{n-j+1}^{*}$, where $w_{j}$ is the $j$ th weight of the DFOWA and $w_{n-j+1}^{*}$ is the $j$ th weight of the AFOWA operator. The FOWA operator is commutative, monotonic, bounded and idempotent. Different families of FOWA operators can be obtained by choosing a different manifestation in the weighting vector, such as the step-FOWA operator, the window-FOWA operator, the FOWA median operator, the S-FOWA and the centered-FOWA operator (Merigó, 2008).

\subsection{Immediate probabilities}

The immediate probability (IP) is a concept that tries to include the decision maker's attitude in a probabilistic decision-making 
problem. We can then represent in the same problem the probabilistic information with the attitudinal character of the decision maker. The main advantage is that it is very easy to use. Therefore, it is not difficult to apply it in almost all the probabilistic problems studied before such as in decision-making problems, actuarial sciences and statistics. The motivation for using it is that the probabilistic information is objective but uncertain. We cannot then guarantee that the expected result is the result that will happen in the future. Due to the fact that we are in a situation of uncertainty (risk environments), each decision maker will have a different attitude regarding the same problem. For example, an optimistic decision maker is more open to consider more risky situations, those that are less certain than the probabilistically expected result. However, a pessimistic decision maker will prefer to consider situations that are safer than the expected result, as determined in calculations with the available probabilistic information.

In order to develop the analysis, we will use in the same formulation the weights of the OWA operator and the probabilistic information. We will refer to it as the IP-OWA operator. It can be defined as follows.

Definition 2. An IP-OWA operator of dimension $n$ is a mapping IP$O W A: R^{n} \rightarrow R$ that has an associated weighting vector $W$ of dimension $n$ such that the sum of the weights is 1 and $w_{j} \in[0,1]$, then:

IP-OWA $\left(a_{1}, a_{2}, \ldots, a_{n}\right)=\sum_{j=1}^{n} \hat{p}_{j} b_{j}$

where $b_{j}$ is the $j$ th largest of the $a_{i}$, each $a_{i}$ has associated a probability $p_{i}, p_{j}$ is the associated probability of $b_{j}$, and $\hat{p}_{j}=\left(w_{j} p_{j} / \sum_{j=1}^{n} w_{j} p_{j}\right)$.

From a generalized perspective of the reordering step, it is possible to distinguish between descending and ascending orders. By using a different manifestation of the weighting vector, it is possible to study different families of IP-OWA operators such as the olympic-IP-OWA, the median-IP-OWA, the centered-IP-OWA and the S-IP-OWA.

\section{Immediate probabilistic fuzzy OWA operator}

The use of information represented in the form of FNs in decision making with immediate probabilities can be useful in situations with high degrees of uncertainty. In these situations, it is not possible to assess the information with exact numbers because we need to consider optimistic and pessimistic results. This problem can be solved by using FNs in the analysis, because they consider a wide range of optimistic and pessimistic results. The main advantage to using FNs is that it provides a more complete view of the uncertain decision problem to the decision maker.

In order to assess this type of problem, we will develop a new aggregation operator: the IP-FOWA operator. It is very similar to the IP-OWA, with the difference being that it can assess the information in a more complete way by using FNs. Then this aggregation operator can assess uncertain information considering a wide range of optimistic and pessimistic results. Moreover, it also uses probabilistic information and the attitudinal character of the decision maker in the same formulation. It can be defined as follows.

Definition 3. Let $\Psi$ be the set of FNs. An IP-FOWA operator of dimension $n$ is a mapping IP-FOWA: $\Psi^{n} \rightarrow \Psi$ that has an associated weighting vector $W$ of dimension $n$ such that the sum of the weights is 1 and $w_{j} \in[0,1]$, then:

IP-FOWA $\left(\tilde{a}_{1}, \tilde{a}_{2}, \ldots, \tilde{a}_{n}\right)=\sum_{j=1}^{n} \hat{p}_{j} b_{j}$, where $b_{j}$ is the $j$ th largest of the $\tilde{a}_{i}$, each $\tilde{a}_{i}$ has associated a probability $p_{i}, p_{j}$ is the probability $p_{i}$ reordered according to $b_{j}$, $\hat{p}_{j}=\left(w_{j} p_{j} / \sum_{j=1}^{n} w_{j} p_{j}\right)$ and the $\tilde{a}_{i}$ are FNs.

Note that we could formulate the whole equation as follows:

$\operatorname{IP-FOWA}\left(\tilde{a}_{1}, \tilde{a}_{2}, \ldots, \tilde{a}_{n}\right)=\sum_{j=1}^{n}\left(\frac{w_{j} p_{j}}{\sum_{j=1}^{n} w_{j} p_{j}}\right) b_{j}$.

Moreover, note that an IP-FOWA operator can also be formulated according to the ordering established with the probabilities $p_{i}$, in the following way.

Definition 4. Let $\Psi$ be the set of FNs. An IP-FOWA operator of dimension $n$ is a mapping IP-FOWA: $\Psi^{n} \rightarrow \Psi$ that has an associated weighting vector $W$ of dimension $n$ such that the sum of the weights is 1 and $w_{j} \in[0,1]$, then:

$\operatorname{IP-FOWA}\left(\tilde{a}_{1}, \tilde{a}_{2}, \ldots, \tilde{a}_{n}\right)=\sum_{j=1}^{n} \hat{p}_{i} \tilde{a}_{i}$,

where $\tilde{a}_{i}$ is the argument variable represented in the form of FNs, each $\tilde{a}_{i}$ has associated a weight $w_{j}$ being $j$ the reordering of the arguments $\tilde{a}_{i}$ in decreasing order, $w_{i}$ is the associated weight $w_{j}$ reordered according to $\tilde{a}_{i}$, and $\hat{p}_{i}=\left(w_{i} p_{i} / \sum_{i=1}^{n} w_{i} p_{i}\right)$.

From a generalized perspective of the reordering step, we can distinguish between the descending IP-FOWA (IP-DFOWA) operator and the ascending IP-FOWA (IP-AFOWA) operator. The weights of these operators are related by $w_{j}=w_{n-j+1}^{*}$, where $w_{j}$ is the $j$ th weight of the IP-DFOWA and $w_{j}=w_{n-j+1}^{*}$ the $j$ th weight of the IPAFOWA operator. As we can see, the main difference is that in the IP-AFOWA operator, the elements $b_{j}(j=1,2, \ldots, n)$ are ordered in an increasing way: $b_{1} \leqslant b_{2} \leqslant \cdots \leqslant b_{n}$ while in the IP-DFOWA, they are ordered in a decreasing way.

Note that the reordering of the arguments (i.e., the establishment of a total order) has an additional difficulty because now we are using FNs; in some cases, it is not clear which FN is higher. We therefore need to establish an additional criterion for ranking FNs. For simplicity, we recommend adherence to the procedure as commented in Merigó (2008), that is, the selection of the result with the highest degree of membership. If this result is an interval, then calculate the average of this interval. However, note that there are a lot of other methods for ranking FNs (Dubois \& Prade, 1980; Kaufmann \& Gupta, 1985; Merigó, 2008). The method used for ranking FNs will depend on the particular problem we are analyzing.

Note also that in a more complicated analysis, it would be possible to consider that the weights $w_{j}$ are themselves also FNs. Another complex situation would be to mix in the same problem information given with interval numbers and information given with FNs.

A further, interesting issue is that it is possible to use a wide range of FNs, such as triangular FNs, trapezoidal FNs, generalized FNs, interval-valued FNs, intuitionistic FNs and even more complex structures (Dubois \& Prade, 1980; Kaufmann \& Gupta, 1985; Merigó, 2008; Merigó \& Casanovas, 2008; Xu \& Yager, 2008; Yager, 2008; Zhou et al., 2008).

If $B$ is a vector corresponding to the ordered arguments $b_{j}$, then we shall call this the ordered argument vector and $W^{T}$ is the transpose of the weighting vector, and the IP-FOWA operator can be expressed as:

$\operatorname{IP-FOWA}\left(\tilde{a}_{1}, \tilde{a}_{2}, \ldots, \tilde{a}_{n}\right)=W^{T} B$.

Note that in the IP-FOWA operator, if the weighting vector is not normalized, i.e., $W=\sum_{j=1}^{n} w_{j} \neq 1$, then the result is still the same because the transformation developed in the construction 
of the immediate probabilities normalizes the results. The same happens if the sum of the weighting vector of the probabilities is not 1 .

Note also that the IP-FOWA operator is bounded and idempotent. These properties can be demonstrated with the following theorems.

Theorem 1 (Idempotency). Assume $f$ is the IP-FOWA operator, if $\tilde{a}_{i}=a$, for all $\tilde{a}_{i}$, then:

$f\left(\tilde{a}_{1}, \tilde{a}_{2}, \ldots, \tilde{a}_{n}\right)=a$

Proof. Since $\tilde{a}_{i}=a$, for all $\tilde{a}_{i}$, we have

$f\left(\tilde{a}_{1}, \tilde{a}_{2}, \ldots, \tilde{a}_{n}\right)=\sum_{j=1}^{n} w_{j} b_{j}=\sum_{j=1}^{n} w_{j} b=b \sum_{j=1}^{n} w_{j}$,

Since $\sum_{j=1}^{n} w_{j}=1$, we get

$f\left(\tilde{a}_{1}, \tilde{a}_{2}, \ldots, \tilde{a}_{n}\right)=a$.

Theorem 2 (Boundedness). Assume $f$ is the IP-FOWA operator then:

$\operatorname{Min}\left\{\tilde{a}_{i}\right\} \leqslant f\left(\tilde{a}_{1}, \tilde{a}_{2}, \ldots, \tilde{a}_{n}\right) \leqslant \operatorname{Max}\left\{\tilde{a}_{i}\right\}$.

Proof. Let $\max \left\{\tilde{a}_{i}\right\}=b$, and $\min \left\{\tilde{a}_{i}\right\}=a$, then

$f\left(\tilde{a}_{1}, \tilde{a}_{2}, \ldots, \tilde{a}_{n}\right)=\sum_{j=1}^{n} w_{j} b_{j} \leqslant \sum_{j=1}^{n} w_{j} b=b \sum_{j=1}^{n} w_{j}$

$f\left(\tilde{a}_{1}, \tilde{a}_{2}, \ldots, \tilde{a}_{n}\right)=\sum_{j=1}^{n} w_{j} b_{j} \geqslant \sum_{j=1}^{n} w_{j} b=b \sum_{j=1}^{n} w_{j}$.

Because $\sum_{j=1}^{n} w_{j}=1$, we obtain

$f\left(\tilde{a}_{1}, \tilde{a}_{2}, \ldots, \tilde{a}_{n}\right) \leqslant b$

$f\left(\tilde{a}_{1}, \tilde{a}_{2}, \ldots, \tilde{a}_{n}\right) \leqslant a$

Therefore,

$\operatorname{Min}\left\{\tilde{a}_{i}\right\} \leqslant f\left(\tilde{a}_{1}, \tilde{a}_{2}, \ldots, \tilde{a}_{n}\right) \leqslant \operatorname{Max}\left\{\tilde{a}_{i}\right\}$.

Note that the IP-FOWA operator is not commutative or monotonic. It is not commutative because the probabilistic aggregation performed by using weighted averages is not commutative. It is not monotonic due to the inconsistencies explained by Liu (2005) about the concept of immediate probability.

Another interesting issue to consider are the measures for characterizing the weighting vector $W=\left(w_{1}, w_{2}, \ldots, w_{n}\right)$ of the IPFOWA operator such as the attitudinal character, the entropy of dispersion, the divergence of $W$ and the balance operator. Note that these measures follow the same methodology as the original version developed for the OWA operator (Yager, 1988) also explained by Merigó (2008).

\section{Families of IP-FOWA operators}

Different types of IP-FOWA operators may be found by using different manifestations of the weighting vector. For example, we can obtain the fuzzy maximum, the fuzzy minimum, the fuzzy average (FA) and the fuzzy weighted average (FWA). Note that these results are found in the weighting vector $W$ prior to the transformation developed in the immediate probability.

Remark 1. The fuzzy maximum is obtained if $w_{1}=1$ and $w_{j}=0$, for all $j \neq 1$.
Remark 2. The fuzzy minimum is obtained if $w_{n}=1$ and $w_{j}=0$, for all $j \neq n$.

Remark 3. More generally, if $w_{k}=1$ and $w_{j}=0$, for all $j \neq k$, we get IP-FOWA $\left(\tilde{a}_{1}, \tilde{a}_{2}, \ldots, \tilde{a}_{n}\right)=b_{k}$, where $b_{k}$ is the $k$ th largest argument $\tilde{a}_{i}$. Note that this type of aggregation is known as the step-IP-FOWA aggregation.

Remark 4. The FA is found when $w_{j}=1 / n$, and $p_{i}=1 / n$, for all $\tilde{a}_{i}$ Note that in the FA, is when we find the usual probabilistic results.

Remark 5. The FWA (or fuzzy probabilistic aggregation) is obtained when $w_{j}=1 / n$, for all $\tilde{a}_{i}$. And the FOWA operator is found when $p_{i}=1 / n$, for all $\tilde{a}_{i}$

Remark 6. Note that the IP-FOWA becomes the usual IP-OWA when the FNs are reduced to the usual exact numbers.

Remark 7. Note that the IP-FOWA becomes the immediate probabilistic uncertain OWA (IP-UOWA) operator when the FNs are reduced to the interval numbers (Moore, 1966). Note that this process is obtained considering only some critical points of the FN such as the maximum, the minimum and the result or results with the highest degree of membership.

Following a similar methodology to those developed in (Amin \& Emrouznejad, 2006; Emrouznejad, 2008; Liu, 2008; Merigó, 2008; Xu, 2005; Yager, 1993, 2007, 2009; Yager \& Filev, 1994), we could study other particular cases of the IP-FOWA operator such as the window-IP-FOWA, the olympic-IP-FOWA, the centered-IP-FOWA operator, the S-IP-FOWA operator, the median-IP-FOWA and the maximal entropy IP-FOWA weights.

Note that the main advantage of using OWA operators is that we can consider a parameterized family of aggregation operators between the maximum and the minimum, depending on the particular interests we have in the problem and its uses. Therefore, these families represent some examples of how to aggregate with the IP-FOWA when considering some particular aspect we want to take into account. Obviously, the selection of the particular method will depend on the particular interests of the decision maker in the given problem.

Note also that the calculation of the OWA weights has been a very popular topic in recent years. Therefore, in the future, we can expect that more methods for determining the OWA weights will appear that can be implemented as different extensions of the OWA operators, such as the IP-FOWA operator developed here.

Remark 8. For example, when $w_{j^{*}}=1 / m$ for $k \leqslant j^{*} \leqslant k+m-1$ and $w_{j^{*}}=0$ for $j^{*}>k+m$ and $j^{*}<k$, we are using the window-IP-FOWA operator. Note that $k$ and $m$ must be positive integers such that $k+m-1 \leqslant n$. Also note that if $m=k=1$, the window-IP-FOWA is transformed in the fuzzy maximum. If $m=1, k=n$, then the window-IP-FOWA becomes the fuzzy minimum. Also, if $m=n$ and $k=1$, then the window-IP-FOWA is transformed in the FA.

Remark 9. The Olympic-IP-FOWA, based on the Olympic average, is found when $w_{1}=w_{n}=0$, and for all others $w_{j^{*}}=1 /(n-2)$. Note that if $n=3$ or $n=4$, then the Olympic-IP-FOWA is transformed in the median-IP-FOWA and if $m=n-2$ and $k=2$, then the window-IP-FOWA is transformed in the Olympic-IP-FOWA.

Remark 10. Another interesting family is the S-IP-FOWA operator It can be subdivided in three classes: the "or-like", the "and-like" and the generalized S-IP-FOWA operator. The generalized S-IPFOWA operator is obtained when $w_{1}=(1 / n)(1-(\alpha+\beta))+\alpha, w_{n}=$ $(1 / n)(1-(\alpha+\beta))+\beta$, and $w_{j}=(1 / n)(1-(\alpha+\beta))$ for $j=2$ to $n-1$, 
where $\alpha, \beta \in[0,1]$ and $\alpha+\beta \leqslant 1$. Note that if $\alpha=0$, then the generalized S-IP-FOWA operator becomes the "and-like" S-IP-FOWA operator, and if $\beta=0$, then it becomes the "or-like" S-IP-FOWA operator. Also, note that if $\alpha+\beta=1$, then we get the IP-fuzzy Hurwicz criteria (IP-FHC).

Remark 11. Note that the median can also be used as IP-FOWA operators. For the median-IP-FOWA, if $n$ is odd, then we assign $w_{(n+1) / 2}=1$ and $w_{j^{*}}=0$ for all others. If $n$ is even, then we assign, for example, $w_{n / 2}=w_{(n / 2)+1}=0.5$ and $w_{j^{*}}=0$ for all others.

Remark 12. For the weighted median-IP-FOWA, we select the argument $b_{k}$ that has the $k$ th largest argument such that the sum of the weights from 1 to $k$ is equal or higher than 0.5 and the sum of the weights from 1 to $k-1$ is less than 0.5 .

Remark 13. Another family of aggregation operator that could be used is the centered-IP-FOWA operator. Following the same methodology as (Yager, 2007), we could define a IP-FOWA operator as a centered aggregation operator if it is symmetric, strongly decaying and inclusive. Note that these properties have to be considered for the weighting vector $w$ of the OWA operator. It is symmetric if $w_{j}=w_{j+n-1}$. It is strongly decaying when $i<j \leqslant(n+1) / 2$ then $w_{i}<w_{j}$ and when $i>j \geqslant(n+1) / 2$ then $w_{i}<w_{j}$. It is inclusive if $w_{j}>0$.

Remark 14. A very useful approach for obtaining the weights, which it is also applicable for the IP-FOWA operator, is the functional method introduced by Yager for the OWA operator. It can be summarized as follows. Let $f$ be a function $f:[0,1] \rightarrow[0,1]$ such that $f(0)=f(1)$ and $f(x) \geqslant f(y)$ for $x>y$. We call this function a basic unit interval monotonic function (BUM). Using this BUM function we obtain the IP-FOWA weights $w_{j}$ for $j=1$ to $n$ as

$w_{j}=f\left(\frac{j}{n}\right)-f\left(\frac{j-1}{n}\right)$.

It can easily be shown that when using this method that $w_{j}$ satisfactorily sum to 1 and each $w_{j} \in[0,1]$.

\section{Illustrative example}

In the following section, we develop an illustrative example about the use of immediate probabilities in fuzzy decision-making problems. We analyze a decision-making problem where a company is studying which strategy is the most appropriate for them. As the environment is very uncertain, the group of experts in the company needs to assess the available information by using FNs. In this example, we will assume that the available information can be assessed with triangular FNs.

We will use different types of FN aggregation operators in order to see that depending on the aggregation operator used, the decision will have different outcomes. We will consider the fuzzy IPmaximum, the fuzzy IP-minimum, the fuzzy IP-average (IP-FA), the fuzzy IP-weighted average (IP-FWA), the IP-FOWA operator, the step-IP-FOWA $(k=2)$, the olympic-IP-FOWA, the median-IPFOWA, the OR-IP-FOWA $(\alpha=0.6)$ and the AND-IP-FOWA $(\beta=0.7)$.

Assume a company that operates in Europe and North America is analyzing its general policy for the next year. The board of directors have analyzed the economic situation of the company and they have found that now is a good moment to make an expansion policy to another continent in order to become more relevant in the world. They consider five possible strategies to follow.

(1) $A_{1}$ : Expand to the Asian market.

(2) $A_{2}$ : Expand to the African market.
(3) $A_{3}$ : Expand to the South American market.

(4) $A_{4}$ : Expand to all three continents.

(5) $A_{5}$ : Do not develop any expansion.

In order to evaluate these strategies, the company considers that the key factor is the economic situation for the next year. Then, depending on the situation, the expected benefits for the company will be different. The experts have considered five possible situations for the next year:

(1) $S_{1}=$ Negative growth rate.

(2) $S_{2}=$ Growth rate near 0 .

(3) $S_{3}=$ Low growth rate.

(4) $S_{4}=$ Medium growth rate.

(5) $S_{5}=$ High growth rate.

Note that it is possible to consider other variables instead of the economic situation but in this example, the board of directors prefers to focus only on this variable in the analysis.

The expected results depending on the situation $S_{i}$ and the alternative $A_{k}$ are shown in Table 1 . Note that the results are triangular FNs.

In this problem, the experts of the company find probabilistic information given as follows: $P=(0.3,0.3,0.2,0.1,0.1)$. Moreover, the policy of the company is to be very pessimistic whenever the future results are not clear. Therefore, they decide to manipulate the probabilities by using the following OWA weighting vector: $W=(0.1,0.2,0.2,0.2,0.3)$. Note that the company will use immediate probabilities in order to assess this problem. The results found in the immediate probabilities by using the above probabilities and weights are shown in Table 2.

Note that we can only use the information shown in Table 2 when using the IP-FOWA operator. Once the initial information is established, it is possible to aggregate it in order to take a decision. First, we will consider some basic aggregation operators, such as the fuzzy IP-maximum, the fuzzy IP-minimum, the IP-FA, the IPFWA and the IP-FOWA operator, to be used in the aggregation. The results are shown in Table 3.

As we can see, the decision is different depending on the aggregation used. If we use the IP-Fuzzy-Minimum, the IP-FA and the IPFWA, then the optimal strategy is $A_{5}$. If we use the IP-Fuzzy-Maximum, then the best choice is $A_{4}$. Also, if we use the IP-FOWA, then the best alternative is $A_{3}$.

Now, we consider the results obtained by using other particular cases of IP-FOWA operators such as the step-IP-FOWA $(k=2)$, the Olympic-IP-FOWA, the median-IP-FOWA, the OR-IP-FOWA

Table 1

Available information about the strategies.

\begin{tabular}{llllll}
\hline & $S_{1}$ & $S_{2}$ & $S_{3}$ & $S_{4}$ & $S_{5}$ \\
\hline$A_{1}$ & $(60,70,80)$ & $(60,70,80)$ & $(50,60,70)$ & $(10,20,30)$ & $(50,60,70)$ \\
$A_{2}$ & $(40,50,60)$ & $(10,20,30)$ & $(80,90,100)$ & $(70,80,90)$ & $(40,50,60)$ \\
$A_{3}$ & $(60,70,80)$ & $(40,50,60)$ & $(60,70,80)$ & $(20,30,40)$ & $(80,90,100)$ \\
$A_{4}$ & $(30,40,50)$ & $(40,50,60)$ & $(60,70,80)$ & $(20,30,40)$ & $(90,100,110)$ \\
$A_{5}$ & $(50,60,70)$ & $(70,80,90)$ & $(30,40,50)$ & $(60,70,80)$ & $(60,70,80)$ \\
\hline
\end{tabular}

Table 2

Immediate probabilities of the problem.

\begin{tabular}{llllll}
\hline & $S_{1}$ & $S_{2}$ & $S_{3}$ & $S_{4}$ & $S_{5}$ \\
\hline $\mathrm{IP}_{1}$ & 0.166 & 0.333 & 0.222 & 0.111 & 0.166 \\
$\mathrm{IP}_{2}$ & 0.095 & 0.095 & 0.285 & 0.095 & 0.428 \\
$\mathrm{IP}_{3}$ & 0.05 & 0.3 & 0.2 & 0.3 & 0.15 \\
$\mathrm{IP}_{4}$ & 0.05 & 0.2 & 0.3 & 0.3 & 0.15 \\
$\mathrm{IP}_{5}$ & 0.157 & 0.105 & 0.105 & 0.315 & 0.315 \\
\hline
\end{tabular}


Table 3

Fuzzy aggregated results 1 .

\begin{tabular}{|c|c|c|c|c|c|}
\hline & IP-Max & IP-Min & IP-FA & IP-FWA & IP-FOWA \\
\hline$A_{1}$ & $(60,70,80)$ & $(10,20,30)$ & $(52,62,72)$ & $(50.5,60.5,70.5)$ & $(48.3,58.3,68.3)$ \\
\hline$A_{2}$ & $(80,90,100)$ & $(10,20,30)$ & $(42,52,62)$ & $(42.2,52.2,62.2)$ & $(33.7,43.7,53.7)$ \\
\hline$A_{3}$ & $(80,90,100)$ & $(20,30,40)$ & $(52,62,72)$ & $(52.2,62.2,72.2)$ & $(49,59,69)$ \\
\hline$A_{4}$ & $(90,100,110)$ & $(20,30,40)$ & $(44,54,64)$ & $(48.8,58.8,68.8)$ & $(40.5,50.5,60.5)$ \\
\hline$A_{5}$ & $(70,80,90)$ & $(30,40,50)$ & $(54,64,74)$ & $(55,65,75)$ & $(48.8,58.8,68.8)$ \\
\hline
\end{tabular}

Table 4

Fuzzy aggregated results 2 .

\begin{tabular}{|c|c|c|c|c|c|}
\hline & IP-Step & IP-Olym & IP-Or-S & IP-And-S & IP-Med \\
\hline$A_{1}$ & $(60,70,80)$ & $(55,65,75)$ & $(56.8,66.8,76.8)$ & $(22.6,32.6,42.6)$ & $(50,60,70)$ \\
\hline$A_{2}$ & $(70,80,90)$ & $(46,56,66)$ & $(64.8,74.8,84.8)$ & $(19.6,29.6,39.6)$ & $(40,50,60)$ \\
\hline$A_{3}$ & $(60,70,80)$ & $(52.5,62.5,72.5)$ & $(68.8,78.8,88.8)$ & $(29.6,39.6,49.6)$ & $(60,70,80)$ \\
\hline$A_{4}$ & $(60,70,80)$ & $(41.2,51.2,61.2)$ & $(71.6,81.6,91.6)$ & $(27.2,37.2,47.2)$ & $(40,50,60)$ \\
\hline$A_{5}$ & $(60,70,80)$ & $(54,64,74)$ & $(63.6,73.6,83.6)$ & $(37.2,47.2,57.2)$ & $(60,70,80)$ \\
\hline
\end{tabular}

$(\alpha=0.6)$ and the AND-IP-FOWA $(\beta=0.7)$. The results are shown in Table 4.

As we can see, in this case, we also arrived at different results, which each depend on the operator used. If we use the IP-StepFOWA, the best choice is $A_{2}$; if we use the IP-Olympic-FOWA, then, $A_{1}$; with the IP-Or-S-FOWA, the $A_{4}$; with the IP-And-S-FOWA, the $A_{5}$; and with the IP-Median-FOWA, the $A_{3}$ and the $A_{5}$.

Note that the results given in the form of triangular FNs can also be represented by using their membership functions. For simplicity, we will simply consider the results shown in Tables 3 and 4.

Another interesting issue is to establish an ordering of the alternatives. This becomes useful when we want to consider more than one alternative. The results are shown in Table 5. Note that $\}$ means "preferred to".

As we can see, depending on the aggregation used, the ordering of the strategies is different. Therefore, depending on the aggregation operator used, the results may lead to different decisions.

Note that in most cases, the reordering is the same for all the aggregation techniques. However, in some cases, when the results between the alternatives are very equal, each particular case of the IP-FOWA (or other types of OWA) may give different orderings because each particular method may fluctuate closer to the minimum or to the maximum. Note also that we prefer to focus on these critical results because we believe that they are more interesting when looking for the main advantages and characteristics of the IPFOWA operator.

\section{Conclusions}

We analyzed the use of FNs in decision making with immediate probabilities. We demonstrated the feasibility of using FNs in this type of problems because it gives a more complete view of the uncertain decision problem. Moreover, by using immediate probabilities, we were able to develop a framework that simultaneously considers the probabilistic information and the attitudinal charac-

Table 5

Ordering of the alternatives.

\begin{tabular}{llll}
\hline \multicolumn{3}{c}{ Ordering } & Ordering \\
\hline IP-FMaximum & $\left.\left.\left.A_{4}\right\} A_{2}=A_{3}\right\} A_{5}\right\} A_{1}$ & IP-Step-FOWA & $\left.A_{2}\right\} A_{1}=A_{3}=A_{4}=A_{5}$ \\
IP-FMinimum & $\left.\left.A_{5}\right\} A_{3}=A_{4}\right\} A_{1}=A_{2}$ & IP-Olym-FOWA & $\left.\left.\left.\left.A_{1}\right\} A_{5}\right\} A_{3}\right\} A_{2}\right\} A_{4}$ \\
IP-FA & $\left.\left.\left.A_{5}\right\} A_{1}=A_{3}\right\} A_{4}\right\} A_{2}$ & IP-Or-S-FOWA & $\left.\left.\left.\left.A_{4}\right\} A_{3}\right\} A_{2}\right\} A_{5}\right\} A_{1}$ \\
IP-FWA & $\left.\left.\left.\left.A_{5}\right\} A_{3}\right\} A_{1}\right\} A_{4}\right\} A_{2}$ & IP-And-S-FOWA & $\left.\left.\left.\left.A_{5}\right\} A_{3}\right\} A_{4}\right\} A_{1}\right\} A_{2}$ \\
IP-FOWA & $\left.\left.\left.\left.A_{3}\right\} A_{5}\right\} A_{1}\right\} A_{4}\right\} A_{2}$ & IP-Med-FOWA & $\left.\left.A_{3}=A_{5}\right\} A_{1}\right\} A_{2}=A_{4}$ \\
\hline
\end{tabular}

ter of the decision maker. In order to develop the analysis, we introduced a new aggregation operator, the IP-FOWA operator. We showed that this operator has similar properties to the OWA operator, the main difference being that it is able to consider probabilities in the problem. We then showed that it is possible to distinguish between descending and ascending orders, study different measures for characterizing the weighting vector, analyze different families of IP-FOWA operators such as the olympic-IP-FOWA or the S-IP-FOWA.

We also developed an application of the new approach in a decision-making problem about selection of strategies. We demonstrated that it is possible to be more optimistic or pessimistic in probabilistic decision-making problems. Obviously, this manipulation of the probabilistic results may give different results that lead to different decisions. Note that there are a lot of other potential applications of this method, which could be developed. Generally, immediate probabilities can be applied in almost all the problems where the usual probabilities have been past employed, such as decision making, actuarial sciences and statistics.

In our future research, we expect to further develop the decision-making problem with immediate probabilities by adding new characteristics in the problem, such as the use of inducing orders or hybrid aggregations. We will also consider other uncertain environments such as the ones that use interval numbers or linguistic variables.

\section{Acknowledgements}

We would like to thank the area editor and the anonymous reviewers for their valuable comments that have improved the quality of the paper.

\section{References}

Alonso, S., Cabrerizo, F. J., Chiclana, F., Herrera, F., \& Herrera-Viedma, E. (2009). Group decision making with incomplete fuzzy linguistic preference relations. International Journal of Intelligent Systems, 24(2), 201-222.

Amin, G. R., \& Emrouznejad, A. (2006). An extended minimax disparity to determine the OWA operator weights. Computers \& Industrial Engineering, 50(3), 312-316.

Beliakov, G., Pradera, A., \& Calvo, T. (2007). Aggregation functions: A guide for practitioners. Berlin: Springer-Verlag.

Canós, L., \& Liern, V. (2008). Soft computing-based aggregation methods for human resource management. European Journal of Operational Research, 189(3), 669-681.

Chang, S. S. L., \& Zadeh, L. A. (1972). On fuzzy mapping and control. IEEE Transactions on Systems, Man and Cybernetics, 2(1), 30-34.

Dubois, D., \& Prade, H. (1980). Fuzzy sets and systems: Theory and applications. New York: Academic Press.

Emrouznejad, A. (2008). MP-OWA: The most preferred OWA operator. KnowledgeBased Systems, 21(8), 847-851. 
Engemann, K. J., Filev, D. P., \& Yager, R. R. (1996). Modelling decision making using immediate probabilities. International Journal of General Systems, 24(3), 281-294.

Engemann, K. J., Miller, H. E., \& Yager, R. R. (2004). Decision making with attitudinal based expected values. International Journal of Technology, Policy and Management, 4(1), 1-12.

Figueira, J., Greco, S., \& Ehrgott, M. (2005). Multiple criteria decision analysis: State of the art surveys. Boston: Springer.

Gil-Aluja, J. (1999). Elements for a theory of decision in uncertainty. Dordrecht: Kluwer Academic Publishers.

Kacprzyk, J., \& Zadrozny, S. (2009). Towards a generalized and unified characterization of individual and collective choice functions under fuzzy and nonfuzzy preferences and majority via ordered weighted average operators. International Journal of Intelligent Systems, 24(1), 4-26.

Kaufmann, A., \& Gupta, M. M. (1985). Introduction to fuzzy arithmetic. Rheinhold: Publications Van Nostrand.

Liu, X. (2005). Preference solutions of probability decision making with RIM quantifiers. International Journal of Intelligent Systems, 20(12), 1253-1271.

Liu, X. (2008). A general model of parameterized OWA aggregation with given orness level. International Journal of Approximate Reasoning, 48(2), 598-627.

Merigó, J. M. (2008). New extensions to the OWA operators and their application in decision making. PhD thesis (in Spanish), Department of Business Administration, University of Barcelona, Spain.

Merigó, J. M., \& Casanovas, M. (2008). Using fuzzy numbers in heavy aggregation operators. International Journal of Information Technology, 4(3), 177-182.

Merigó, J. M., \& Casanovas, M. (2009). Induced aggregation operators in decision making with the Dempster-Shafer belief structure. International Journal of Intelligent Systems, 24(8), 934-954.

Merigó, J. M., \& Gil-Lafuente, A. M. (2008). The generalized adequacy coefficient and its application in strategic decision making. Fuzzy Economic Review, 13(2), 17-36.

Merigó, J. M., \& Gil-Lafuente, A. M. (2009). The induced generalized OWA operator. Information Sciences, 179(6), 729-741.

Mitchell, H. B., \& Estrakh, D. D. (1998). An OWA operator with fuzzy ranks. International Journal of Intelligent Systems, 13(1), 69-81.

Moore, R. E. (1966). Interval analysis. Englewood Cliffs, NJ: Prentice Hall.

Torra, V. (1997). The weighted OWA operator. International Journal of Intelligent Systems, 12(2), 153-166.

Wang, Y. M., Luo, Y., \& Liu, X. (2007). Two new models for determining OWA operator weights. Computers \& Industrial Engineering, 52(2), 203-209.
Wu, J., Li, J. C., Li, H., \& Duan, W. Q. (2009). The induced continuous ordered weighted geometric operators and their application in group decision making. Computers \& Industrial Engineering, 56(4), 1545-1552.

$\mathrm{Xu}$, Z. S. (2005). An overview of methods for determining OWA weights. International Journal of Intelligent Systems, 20(8), 843-865.

$\mathrm{Xu}, \mathrm{Z}$. S. (2009). An automatic approach to reaching consensus in multiple attribute group decision making. Computers \& Industrial Engineering, 56(4), 1369-1374.

Xu, Z. S., \& Yager, R. R. (2008). Dynamic intuitionistic fuzzy multi-attribute decision making. International Journal of Approximate Reasoning, 48(1), 246-262.

Yager, R. R. (1988). On ordered weighted averaging aggregation operators in multicriteria decision making. IEEE Transactions on Systems, Man and Cybernetics B, $18(1), 183-190$.

Yager, R. R. (1993). Families of OWA operators. Fuzzy Sets and Systems, 59(2), $125-148$.

Yager, R. R. (1999). Including decision attitude in probabilistic decision making. International Journal of Approximate Reasoning, 21(1), 1-21.

Yager, R. R. (2007). Centered OWA operators. Soft Computing, 11(7), 631-639.

Yager, R. R. (2008). Using trapezoids for representing granular objects: Applications to learning and OWA aggregation. Information Sciences, 178(2), 363-380.

Yager, R. R. (2009). Weighted maximum entropy OWA aggregation with applications to decision making under risk. IEEE Transactions on Systems, Man and Cybernetics A, 39(3), 555-564.

Yager, R. R., Engemann, K. J., \& Filev, D. P. (1995). On the concept of immediate probabilities. International Journal of Intelligent Systems, 10(4), 373-397.

Yager, R. R., \& Filev, D. P. (1994). Parameterized and like and or like OWA operators. International Journal of General Systems, 22(3), 297-316.

Yager, R. R., \& Kacprzyk, J. (1997). The ordered weighted averaging operators: Theory and applications. Norwell, MA: Kluwer Academic Publishers.

Zadeh, L. A. (1965). Fuzzy sets. Information and Control, 8(3), 338-353.

Zadeh, L. A. (1975). The concept of a linguistic variable and its application to approximate reasoning. Part 1. Information Sciences, 8(3), 199-249; Part 2. Information Sciences, 8(4), 301-357; Part 3. Information Sciences, 9(1), 4380.

Zarghami, M., \& Szidarovszky, F. (2009). Revising the OWA operator for fuzzy stochastic multi criteria decision making. European Journal of Operational Research, 198(1), 259-265.

Zhou, S. M., Chiclana, F., John, R. I., \& Garibaldi, J. M. (2008). Type-1 OWA operators for aggregating uncertain information with uncertain weights induced by type2 linguistic quantifiers. Fuzzy Sets and Systems, 159(24), 3281-3296. 